\title{
Hyperekplexia-associated mutations in the neuronal glycine transporter 2
}

\author{
Beatriz López-Corcuera ${ }^{\mathrm{a}, \mathrm{b}, *}$, Esther Arribas-González ${ }^{\mathrm{a}, \mathrm{b}}$, Carmen Aragón ${ }^{\mathrm{a}, \mathrm{b}}$ \\ a Centro de Biología Molecular "Severo Ochoa", Universidad Autónoma de Madrid-Consejo Superior de Investigaciones Científicas, Madrid, Spain \\ ${ }^{\mathrm{b}}$ IdiPAZ-Hospital Universitario La Paz, Universidad Autónoma de Madrid, Madrid, Spain
}

A R T I C L E I N F O

\section{Keywords:}

Hyperekplexia

Glycine transporter

GlyT2

Structure

Intracellular trafficking

Dominant mutation

\begin{abstract}
A B S T R A C T
Hyperekplexia or startle disease is a dysfunction of inhibitory glycinergic neurotransmission characterized by an exaggerated startle in response to trivial tactile or acoustic stimuli. Although rare, this disorder can have serious consequences, including sudden infant death. One of the most frequent causes of hyperekplexia are mutations in the SLC6A5 gene, encoding the neuronal glycine transporter 2 (GlyT2), a key component of inhibitory glycinergic presynapses involved in synaptic glycine recycling though sodium and chloride-dependent co-transport. Most GlyT2 mutations detected so far are recessive, but two dominant missense mutations have been described. The detailed analysis of these mutations has revealed structural cues on the quaternary structure of GlyT2, and opens the possibility that novel selective pharmacochaperones have potential therapeutic effects in hyperekplexia.
\end{abstract}

\section{Introduction}

Hyperekplexia or "startle disease" (OMIM 149400), is a rare neurological syndrome characterized by pathological alteration of the brainstem startle reflex controlled by glycinergic neurons. Newborns display neonatal violent spasms, exaggerated trunk and limb stiffness and frequent tremor attacks in response to unexpected although trivial noise or touch stimuli (Hyperekplexia et al., 2007). The disorder is also called "stiff baby syndrome" since the neonatal hypertonia due to impaired motor control precludes the children from executing voluntary movements. Infants with hyperekplexia have swallowing difficulties, weaning complications, and are at risk of brain damage or even sudden death due to apnea episodes unless they are assisted through the Vigevano maneuver, which involves flexing of the head and limbs toward the trunk (Vigevano et al., 1989). Although symptoms often ameliorate during the first year of life, the exaggerated startle response can persist into adulthood and the habituation to the startle, or attenuation of the response after repeated stimulation, is poor or null (Bakker et al., 2006). Adults suffer disabling motor alterations and recurrent unprotected falls throughout their entire life (Andermann et al., 1980). There is not specific treatment for hyperekplexia and the therapy is directed to prevent hypertonia and startle by potentiating inhibitory $\mathrm{GABA}_{\mathrm{A}}$ receptor function with benzodiazepines (Tijssen et al., 1997). Clonazepam is efficacious against startle, although it is not free of side effects such as sedation (Thomas et al., 2013).

Brain stem and spinal cord glycinergic pathways control muscle tone, motor rhythms, spinal reflex responses, and sensory signals (van den Pol and Gorcs, 1988). The startle reflex produces a brief movement similar to that of a shock that involves pairs of antagonistic muscles of the arms, neck, trunk, elbows, hips and knees (Bakker et al., 2006). This is controlled by brain stem glycinergic interneurons mediating circuits of reciprocal inhibition that coordinate the contraction and relaxation of agonist and antagonistic muscle pairs. Inhibitory glycinergic inputs also regulate motoneuron excitability in the adult and during development (Fogarty et al., 2016; Legendre, 2001). Besides, glycine is involved in the processing of auditory information in the cochlear nuclei, in the complex of the superior olive and in the inferior colliculus (Wenthold et al., 1987). Inhibitory glycinergic neurons are involved in the processing of visual information in the retina (Pourcho and Goebel, 1985). In the dorsal horn of the spinal cord, glycine inhibition enables the suppression of nociceptive signals towards superior brain areas therefore controlling nociceptive signal transmission (Foster et al., 2015; Imlach, 2017). The crucial role of glycinergic transmission in human pain processing has been recently confirmed by the finding that patients with diagnosed hyperekplexia have increased pain sensitivity and impaired central pain modulation as compared with control individuals (Vuilleumier et al., 2018).

At the molecular level, hyperekplexia is a glycinergic synaptopathy and affects proteins necessary for the proper functioning of the fast glycine-mediated inhibitory neurotransmission. Glycine released by glycinergic interneurons activates strychnine-sensitive glycine receptors (GlyR), which permit chloride influx through the postsynaptic

\footnotetext{
* Corresponding author. Departamento de Biología Molecular, Centro de Biología Molecular "Severo Ochoa”, C/ Nicolás Cabrera 1, Universidad Autónoma de Madrid, 28049, Madrid, Spain.

E-mail address: blopez@cbm.csic.es (B. López-Corcuera).
} 
membrane leading to hyperpolarization and impairing the propagation of excitatory postsynaptic potentials (Legendre, 2001; Lynch, 2004). GlyRs are ligand-gated heteropentameric receptors that in postnatal life are mainly formed by two $\alpha 1$ channel-forming subunits and three structural $\beta$ subunits (Zeilhofer et al., 2018). Within about 250 cases of hyperekplexia reported in the literature, the most frequent causes are mutations preventing the function of the glycine receptor affecting mainly to the $\alpha 1$ subunits (human GLRA1 gene), and with much lower frequencies to other components of the glycinergic postsynaptic element: $\beta$ subunits (GLRB), collybistin (ARHGEF9) and gephyrin (GPHN), proteins involved in the postsynaptic organization of glycine receptors (Harvey et al., 2008).

\section{The glycine transporter GlyT2}

The second most common causes of hyperekplexia are mutations in the SLC6A5 gene, encoding the presynaptic glycine transporter GlyT2 (Thomas et al., 2013). The neuronal GlyT2 is a key component of inhibitory glycinergic synapses that removes glycine from the synaptic cleft contributing to the termination of the glycinergic signal together with its glial counterpart GlyT1 (Aragon and Lopez-Corcuera, 2003). GlyT2 performs tight $\mathrm{Na}^{+}$-and $\mathrm{Cl}^{-}$-coupled high-affinity glycine cotransport with a stoichiometry of $3 \mathrm{Na}^{+} / 1 \mathrm{Cl}^{-} / 1$ glycine thereby building a stepped gradient of neurotransmitter through the presynaptic plasma membrane (Aragon and Lopez-Corcuera, 2005; Roux and Supplisson, 2000). As revealed by gene inactivation studies in mice, loss of GlyT2 function precludes the refilling of synaptic vesicles with glycine since GlyT2 activity is crucial for supplying substrate to the lowaffinity vesicular glycine transporter VIAAT and hence to preserve quantal glycine content in synaptic vesicles (Gomeza et al., 2003). The phenotype of the knock out mice for the GlyT2 gene recapitulated some of the symptoms of human hyperekplexia including spasticity, rigid muscle tone, strong spontaneous tremor and a severely impaired righting reflex and was lethal during the second postnatal week (Gomeza et al., 2003). These neurological alterations are similar to those displayed by the spastic, spasmodic and oscillator mouse models containing mutations in the GlyR $\alpha 1$ subunit gene (Harvey and Yee, 2013). Recently, a spontaneous retrotransposon insertion in mouse Slc6a5 gene has been shown to cause a related complex phenotype with handling-induced spasms also lethal within two weeks (Bogdanik et al., 2012). Together with these evidences, genetic analyses in hyperekplexia patients revealed mutations in the human GlyT2 gene, present in the short arm of chromosome 11 (Chr 11p15.1-11p15.2) (Morrow et al., 1998), as the cause of a presynaptic form of the disease (Rees et al., 2006). There are currently about 30 mutations affecting human GlyT2 gene (Carta et al., 2012; Eulenburg et al., 2006; Gimenez et al., 2012; Rees et al., 2006) and several have been found in related congenital neurological disorders in dogs (Gill et al., 2011) and cattle (Charlier et al., 2008). Human SLC6A5 is presently the second major gene involved in hyperekplexia showing a frequency of mutations of up to $25 \%$. A recent study showed that patients with GlyT2 gene mutations were significantly more likely to have recurrent infantile apneas and developmental delay than those containing GLRA1 mutations (Thomas et al., 2013).

The SLC6A5 product (GlyT2) belongs to the SLC6 transporter family that encompasses secondary active transporters energized by the $\mathrm{Na}^{+}$ gradient maintained by the $\mathrm{Na}^{+} / \mathrm{K}^{+}$ATPase, a protein that is physically and functionally associated with GlyT2 (de Juan-Sanz et al., 2013b). $\mathrm{Cl}^{-}$is also required for the activity of the eukaryotic family members. Other SLC6 components include GlyT1, the GABA, and the monoamine transporters (Broer and Gether, 2012; Drew and Boudker, 2016; Rudnick et al., 2014). SLC6 proteins contain twelve transmembrane domains (TM) connected by external (EL) and internal loops (IL) and cytoplasmic amino and carboxyl-termini. The folding pattern of the SLC6 transporters was first described in the prokaryotic homologue LeuTAa (leucine transporter from Aquifex aeolicus) (Yamashita et al.,
2005), and later in the eukaryotic crystals, the Drosophila melanogaster dopamine transporter (dDAT) (Penmatsa et al., 2013) and the human serotonin transporter ( $h$ SERT) (Coleman et al., 2016). The transporter TMs shape a cylinder-like structure embedded in the membrane with central substrate and ion binding sites formed with the contribution of partially unwound central portions of TM1 and TM6. The main feature of this folding consists in two topologically inverted structural repeats of five TMs each, which intertwine to form two four-TM bundles: one mobile bundle (core) and a more static bundle (scaffold) (Rudnick et al., 2014). During the transport cycle, the protein binds the substrate and ions in one side of the membrane and undergoes conformational changes that let the two bundles rock one against the other releasing substrate and ions to the opposite side (Jardetzky, 1966). LeuTAa translocation cycle requires two sodium ions. The two sodium-binding sites are conserved within the SLC6 family and some of their functional roles were unveiled: tight substrate coupling for Na1 (Penmatsa and Gouaux, 2014), and stabilization of the outward-facing conformation for Na2 (Claxton et al., 2010).

Based on the bacterial LeuTAa structure, 3D models for glycine transporters were generated and molecular dynamics simulations and mutant analysis confirmed the conservation of the two sodium sites (Perez-Siles et al., 2011). By studying the differential sensitivity of GlyT2 and GlyT1 to lithium ion it was found that Na1 is a high affinity, high selectivity site whereas $\mathrm{Na} 2$ is a lower affinity, lower selectivity site where lithium can bind (Perez-Siles et al., 2011, 2012). Recently, the location of the third sodium site in GlyT2 was proposed and the features of this site and how it is coupled to energize the transport of glycine awaits further investigation (Subramanian et al., 2016). An additional sodium-binding region present in the extracellular vestibule of GlyT2 but absent in GlyT1 was identified indicating a role in cation guiding of the outward-facing cavity of GlyT2 (Perez-Siles et al., 2012; Zomot et al., 2015). The crystal structure of the two eukaryotic SLC6 members solved so far, $d \mathrm{DAT}$ and $h$ SERT, showed differences with the prokaryotic model (Coleman et al., 2016; Penmatsa et al., 2013). The presence of bound cholesterol in both crystals, although in different locations, was particularly relevant for a lipid-raft associated transporter as GlyT2 (Coleman et al., 2016; Nunez et al., 2008; Penmatsa et al., 2013). Recently, a cholesterol site involving TM1a, TM5 and TM7 able to immobilize the cytoplasmic portion of TM5 and to stabilize the transporter in an outward-facing conformation was found to be the preferred binding site for some SLC6 proteins (Zeppelin et al., 2018). The eukaryotic structures gave clues for the first time on the arrangement of large external loops: EL2 and EL4, which may provide substantial information for the binding site of some GlyT2 inhibitors (Vandenberg et al., 2016). An interaction of the carboxyl-terminal helix and the IL1-TM1a essential for the folding and the function of the transporters was also evidenced (El-Kasaby et al., 2010; Penmatsa et al., 2013). Some novel hints on the eukaryote dimerization interface arise from the differential conformation of the TM12 where a proline residue makes the C-terminal half of the helix to protrude outside the monomer (Coleman and Gouaux, 2018). Presently, GlyT2 models based in dDAT have being developed, and will help to investigate selected missense hyperekplexia-associated mutations on critical residues involved in transporter function or trafficking (Carland et al., 2018).

\section{GlyT2 biogenesis}

The synthesis of GlyT2 takes place in ribosomes associated to the endoplasmic reticulum (ER). The ER is the organelle where proteins are folded and maturate under a stringent quality control that warrants ER emergent molecules have the proper structure (Ellgaard and Helenius, 2003). Membrane protein synthesis begins with the co-translational translocation of the nascent peptide to the ER membrane and its lateral inclusion in the membrane in a correct topological orientation (Guna and Hegde, 2018). The ER lumen contains optimal composition: oxidizing conditions, available ATP, $\mathrm{pH}$ neutral, high concentration of 
calcium and optimized ionic environment for protein folding, disulfide bond formation and $\mathrm{N}$-glycosylation. Besides, RE lumen is filled with molecular chaperones, thiol oxidoreductases, prolyl isomerases, and $\mathrm{N}$ linked glycan-modifying enzymes and will include emergent protein domains destined to the extracellular milieu, including $N$-glycosilated regions (Barlowe and Helenius, 2016). Four asparagines in GlyT2 EL2 receive preassembled glycan chains generating an immature underglycosylated GlyT2 precursor that will acquire complex $N$-glycosylation along the secretory pathway. The glycosylation helps to stabilize protein folding permitting the acquisition of the desired biophysical properties (Martinez-Maza et al., 2001; Nunez and Aragon, 1994). Other posttranslational modifications such as acylation may also be inserted in the ER (Shipston, 2014). Correct transporter folding requires interaction with several ER chaperones, some of which recognize and shield hydrophobic TMs to assist folding such as the lectin calnexin (CNX). The biogenesis of the transporter in cells expressing GlyT2 can be modulated by the concentrations of this chaperone that controls the quality of the immature GlyT2 precursor and therefore the amount of active transporter. In cells expressing GlyT2, transporter accumulation and transport activity were attenuated by siRNA-mediated CNX knockdown and enhanced by CNX overexpression (Arribas-Gonzalez et al., 2013). The transient binding of CNX to the immature GlyT2 in the $\mathrm{RE}$ is mediated by glycan and polypeptide-based interactions through which the chaperone discriminates between different GlyT2 folding states. In this way, the sequential site directed removal of selected $\mathrm{N}$ glycans although reduced chaperone binding also increased transporter unfolding. These conditions exert opposite actions on the binding to the chaperone so that a GlyT2 mutant lacking all four $N$-glycan acceptor sites, which exhibits anomalous folding, has also long lasting interaction with CNX. The highest contribution to transporter misfolding takes place after mutagenesis of Asn-366, suggesting high stabilizing role for the glycan linked to Asn-366. Moreover, the modest glycine transport of the mutant lacking all four $N$-glycan acceptor sites could be rescued by CNX overexpression to a higher level than its plasma membrane expression indicating CNX can exert genuine chaperone activity on GlyT2 independent of its lectin ability.

For ER exit, proteins are loaded into COPII transport vesicles that assemble in ER exit sites (ERES). The COPII coat complex contains an inner shell (Sec23/Sec24 complex) that sorts cargoes and an outer cage (Sec13/Sec31 complex) that leads to coat polymerization and induces membrane curvature for budding (Miller et al., 2003). The four Sec24 (A, B, C, D) variants are mainly responsible for efficient cargo loading into the vesicles by binding either directly or through adaptors to export signals and motifs in fully folded and oligomerized proteins. These motifs may not be available until folding and assembly are completed. Incompletely folded cargo proteins are actively removed from ERES (Barlowe and Helenius, 2016). The SLC6 transporters contain an ER export signal in the carboxyl terminus that supports the binding to the Sec24 adaptor (Arribas-Gonzalez et al., 2015; Farhan et al., 2004). GlyT2 seems to share a RL (X8)D motif in the C-terminus with the related GlyT1 that may support the interaction with Sec24D, although additional export signals may be involved (Fernandez-Sanchez et al., 2008). In GlyT2 the removal or mutagenesis of this region prevents ER export and Sec24D interaction (Arribas-Gonzalez et al., 2015; Fernandez-Sanchez et al., 2008). The RL motif is also conserved in GAT1 and it mediates the binding to Sec24D and transporter concentrative ER export (Farhan et al., 2007). Although GAT1 oligomeric assembly seems not to be required for the interaction with Sec24D, oligomerization of transporters may bring together several ER COPIIbinding motifs, thereby increasing the efficiency of ER export. Thus, the "oligomerization hypothesis" for SLC6 transporters claims oligomerization supports efficient recruitment of COPII components and therefore is required for ER export (Farhan et al., 2007; Sitte and Freissmuth, 2003) (Freissmuth et al., 2018). CNX assistance to GlyT2 folding suggests GlyT2 bound to CNX is in a monomeric state and CNX release permits the transporters to form oligomers that can be concentrated in
ERES by interaction with Sec24. The in vivo GlyT2 oligomeric assembly is presently unknown. Previous data in the literature found GlyT2 dimers were generated under nonnative conditions after transporter crosslinking (Bartholomaus et al., 2008). However, it was after the discovery of GlyT2 dominant hyperekplexia mutations that robust evidences for the in vivo occurrence of GlyT2 oligomers were obtained (see below) (Arribas-Gonzalez et al., 2015; Gimenez et al., 2012).

In neurons, the mature GlyT2 transporter traffics to the plasma membrane incorporated into small vesicles containing synaptic vesicle protein components such as synaptophysin, or the vesicular inhibitory amino acid transporter VIAAT but no other protein involved in GABAergic transmission (Nunez et al., 2009). The transporter protein is concentrated at presynaptic elements by interaction with syntenin-1 through a PDZ motif located in GlyT2 C-terminal end (Armsen et al., 2007). GlyT2 is delivered into neuronal plasma membrane lipid-rafts, whose components cholesterol and sphingolipids provide the most favorable environment for optimal transport function (Nunez et al., 2008). Membrane lipids are also stringent regulators of functional interactions of GlyT2 with other plasma membrane transporters such as the neuronal isoforms of $\mathrm{Na}^{+} / \mathrm{K}^{+}$-ATPase (de Juan-Sanz et al., 2013b), the neuronal plasma membrane $\mathrm{Ca}^{2+}$-ATPase (PMCA), and the $\mathrm{Na}^{+}$/ $\mathrm{Ca}^{2+}$-exchanger 1 (NCX1) (de Juan-Sanz et al., 2014). The activities of these transporters are necessary for optimal GlyT2 activity in the presynapse and this modulation depends on lipid raft integrity. These interacting partners are included in a functional unit proposed to maintain local cation gradient homeostasis after neuronal (and GlyT2) intense activity. GlyT2 endocytosis is dependent on ubiquitination of Cterminal lysines, what tags the transporter either for constitutive recycling into Rab11-containing vesicles or for lysosomal degradation (de Juan-Sanz et al., 2011, 2013a). Modulation of GlyT2 trafficking is achieved by several signals in a process also dependent on GlyT2 ubiquitination (de Juan-Sanz et al., 2011).

\section{GlyT2 hyperekplexia-associated mutations}

Several structural and functional properties of GlyT2 have been reported to be altered or could be potentially affected in hyperekplexia patients. Although the majority of GlyT2 mutations associated to hyperekplexia are loss of function and may reduce the glycine supply to the presynaptic terminal impairing the glycinergic neurotransmission, there are GlyT2 mutants that maintain residual or even significant activity and could affect other aspects of GlyT2 physiology. A high proportion of changes found in GlyT2 are nonsense and frameshift mutations (W151X, R191X, Y297X, Y377X, R439X, V432F + fs97, Q630X, P108L + fs25, L198R + fs123, S489F + fs39, I665K + fs1), which truncate the transporters and cause retention of the misfolded inactive protein in the ER. Others are missense mutations affecting residues with crucial roles for the catalytic transport activity such as the sodium binding sites (N509S, A275T), the $\mathrm{Cl}^{-}$site (S513I), or the glycine site (W482R). Some are proposed to affect conformational changes needed for the translocation cycle (L237P, L243T, T425M, Y491C, F547S, Y656H, G657A) (Carta et al., 2012; Eulenburg et al., 2006; Rees et al., 2006). The inheritance of GlyT2 mutations so far found in hyperekplexia patients is mostly recessive or in compound heterozygous state. Though, two dominant missense mutations have been described, whose pathogenic mechanisms have been studied by our group. One of such mutations was found in eight patients from Spain and the United Kingdom after sequencing the 16 exons of the SLC6A5 gene of 204 individuals who had tested negative for mutations in GLRA1 (5q33.1) and GLRB (4q32.1) (Gimenez et al., 2012). A single nucleotide change in exon 15 was found in heterozygosis that caused dominant inheritance and significant variation in clinical presentation. The change introduced a cysteine in TM11, in the outer face of the protein, instead of a conserved tyrosine residue (Y705C). The mutant expressed in heterologous cells and primary neurons was active, and maintained about $60 \%$ glycine transport and surface expression that could be 
rescued by treatment with reducing agents. By mutagenesis of several endogenous cysteine residues in the Y705C background and systematic assay of reducing agent rescue, it was found that the introduced cysteine aberrantly interacts and disrupts an endogenous disulfide bond between Cys-311 and Cys-320 in EL2 of GlyT2. According to the relevant role of this loop in transporter structure and function recently confirmed in the hSERT crystal (Coleman et al., 2016), this interference hinders transporter progression to the plasma membrane and inhibits glycine transport. It is presently unknown whether this feature is related to the dominant inheritance of the mutation since it is recessive in heterologous cells. However, in the same heterologous system the mutant shows altered sensitivity to protons and zinc that appears even when wild-type and mutant are co-expressed, suggesting it is dominant in heterozygosity. Accordingly, the substitution of Y705 by an acidic amino acid such as glutamate or aspartate strongly impaired GlyT2 transport activity. The more acidic character of cysteine as compared to tyrosine might trigger the dysregulation. Further analysis in neural preparations may unveil to which extent this altered regulation impairs glycinergic neurotransmission (Chen et al., 2004; Hirzel et al., 2006; Krishtal et al., 1987).

There is another dominant missense mutation found in hyperekplexia patients that substitutes a serine in TM7 by an arginine (S510R) what prevents mutant maturation (Rees et al., 2006). The mutant blocks the arrival of the transporter to the surface exerting a dominant-negative effect that impairs GlyT2 intracellular trafficking. An extensive analysis of the pathogenic mechanisms of this mutant using the rat sequence, S512R (equivalent to the human mutation $\mathrm{S} 510 \mathrm{R}$ ), was performed and it was found it is a folding-defective mutant that does not produce a mature transporter but rather the immature transporter is retained in the ER (Arribas-Gonzalez et al., 2015). The presence of an arginine but not the removal of serine- 512 provokes the misfolding as proven by multiple mutagenesis analysis in this and contiguous positions including serine-513, a residue substituted by an isoleucine in some hyperekplexia patients with recessive inheritance. The glycosidase sensitivity of S512R indicates mutant maturation is arrested after receiving the first preassembled glycans. Co-translationally trimmed glycans by ER glucosidases generate monoglucosylated chains that support binding to CNX (Hammond et al., 1994). Therefore, S512R is a preferred substrate for the chaperone and has enhanced association to CNX as compared to wild type. In addition, the interaction of the mutant with the adaptor protein Sec24D, responsible for COPII vesicle cargo selection is poor and this impedes mutant exit from the ER. S512R mutant subunits form heteroligomers with wild-type GlyT2. Since the stability of these mixed oligomers is higher than GlyT2 homoligomers, wild-type is retained in the ER and the mutant exerts a dominant-negative effect on GlyT2 traffic. Interestingly, CNX overexpression can rescue the dominant-negative effect by increasing the amount of wild-type GlyT2 that reaches the plasma membrane and attenuating the interaction between wild-type and mutant transporters. Reminiscent of the chaperoning function of proteinaceous chaperones as CNX, the effect of chemical chaperones in the rescue of GlyT2 was analyzed. Several nonselective compounds belonging to the main classes of chemical chaperones were proven and some were able to rescue the wild-type transporter from the dominant negative effect of S512R. The FDA approved compound 4-phenylbutyrate (PBA) promoted significant rescue comparable to that of CNX. It restored both membrane expression and glycine transport of the active GlyT2 co-expressed with the mutant in heterologous cells and primary neurons. PBA is an interesting compound since it can pass across the blood-brain barrier and it has been implicated as protective agent in some neural diseases (Ozcan et al., 2009). It is proposed that the overexpression of CNX or the treatment with PBA can dissociate the wild-type-mutant heteroligomers retained in the ER and this would send the mutant to ER-associated degradation and permit wild-type molecules to reach the plasma membrane and relieve the dominant-negative effect. These results support the possibility that novel selective pharmacopherones may have potential therapeutic effects in hyperekplexia patients. More specific pharmacopherones may be useful in hyperekplexia not only for the treatment of newborns holding GlyT2 mutations affecting transporter trafficking but also to help the relief of the increased pain sensation and impaired central pain modulation the patients with diagnosed hyperekplexia have as compared with control individuals. This may importantly enlarge the usefulness of the obtained medicines since they could be used during lifetime by the adult hyperekplexia patients and improve their quality of life.

The discovery of GlyT2 dominant hyperekplexia mutations has provided robust evidences for the in vivo occurrence of GlyT2 oligomers (Arribas-Gonzalez et al., 2015; Gimenez et al., 2012). The pathogenic mechanisms of the two dominant mutations found in hyperekplexia patients rely on the formation of heteroligomers carrying wild type and mutant protomers. Therefore they are the first in vivo evidence of the quaternary structure of GlyT2. The dominant-negative effect of GlyT2 trafficking exerted by S510R mutant is due to the formation of heteroligomers in the ER. Although this was formerly accepted to be a general requisite for the incorporation into COPII vesicles and the ER exit of SLC6 transporters ("the oligomerization hypothesis", (Sitte and Freissmuth, 2003), this was not previously demonstrated for GlyT2. The arrest exerted by S512R on GlyT2 trafficking is dependent, as expected, on the dose of the mutant co-expressed with the wild-type and can be quantified assuming the basic element of the transporter quaternary structure is a dimer (Coleman et al., 2016; Penmatsa et al., 2013). In SERT, it has been proposed that oligomer formation is initiated in the ER but the ER oligomers are mobile and can exchange protomers in contrast to the plasma membrane oligomers, which are fixed due to the association with phosphatidylinositol-4,5-bisphosphate/PIP2 (Anderluh et al., 2017). For the dominant-negative action of S512R, the remaining transport activity detected when wild-type and mutant were co-expressed was higher than expected assuming all the dimers containing mutant protomers -alone or in combination-were inactive (retained) (Arribas-Gonzalez et al., 2015). The stochastic association of wild-type and S512R mutant would result in residual transport activity of about $25 \%$ assuming all wild-type molecules that interact with S512R are retained (Fig. 1). Higher order oligomers would yield more depressed residual transport activity (i.e. about $4.5 \%$ if it would be a tetramer). This condition was described for the coexpression of wild-type norepinephrine transporter NET and a dominant negative mutant found in familial orthostatic intolerance (Farhan et al., 2006; Hahn et al., 2003). However, the measured residual transport activity of wild-type GlyT2 and S512R was around $45-50 \%$. Since the actual activity of the wild-type/S512R heteromers is not known, this level of residual activity could be reached assuming two possible scenarios: 1) the S512R mutation is not dominant and wild-type/S512R mutants are $50 \%$ active. About $40 \%$ predicted activity could also be obtained assuming tetrameric structure of $50 \%$ active dimers. These conditions are very unlucky as we determined the interaction strength of wild-type/ S512R heteromers is higher than that of wild-type homomers supporting a dominant-negative action of the mutant. 2) The second conjecture that can be posed to explain $45-50 \%$ residual activity for the coexpression is the wild-type/S512R heteromers display glycine transport below $50 \%$ but above $25 \%$. This could be compatible with a nonpermanent arrest of wild-type GlyT2 due to S512R association that can be relieved through monomer exchange. This agrees with a mobile fraction of oligomers in the ER as proposed (Anderluh et al., 2017). Furthermore, the rescue of the dominant-negative effect by CNX overexpression or PBA treatment seems more easily explained with a mobile fraction of ER oligomers. On the other hand, the dominance of the Y705C mutant suggests GlyT2 heteroligomers carrying wild type and mutant protomers reach the plasma membrane. The phenotype of the transporters co-expressed in COS7 cells seems to be quantitatively similar to the phenotype of the Y705C mutant expressed by itself, and presumably forming homoligomers what may sustain the hypothesis of fixed oligomers at the plasma membrane. The investigation of GlyT2 
A

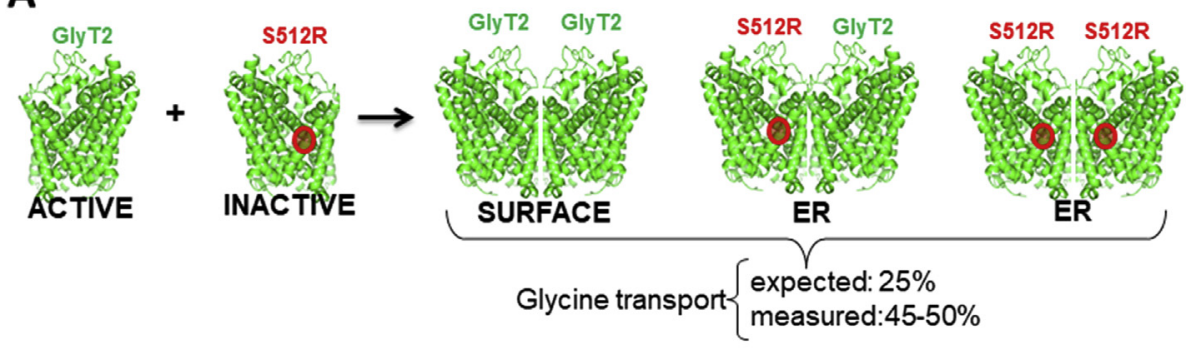

Fig. 1. Schematic diagram of how the hyperekplexia mutations alter expression levels of GlyT2. (A) Predicted and measured glycine transport of wildtype and S512R coexpression. (B) Predicted and measured glycine transport of wild-type and Y705C coexpression after (upper line) or before (lower line) incubation with dithiothreitol (DTT).

\section{B}
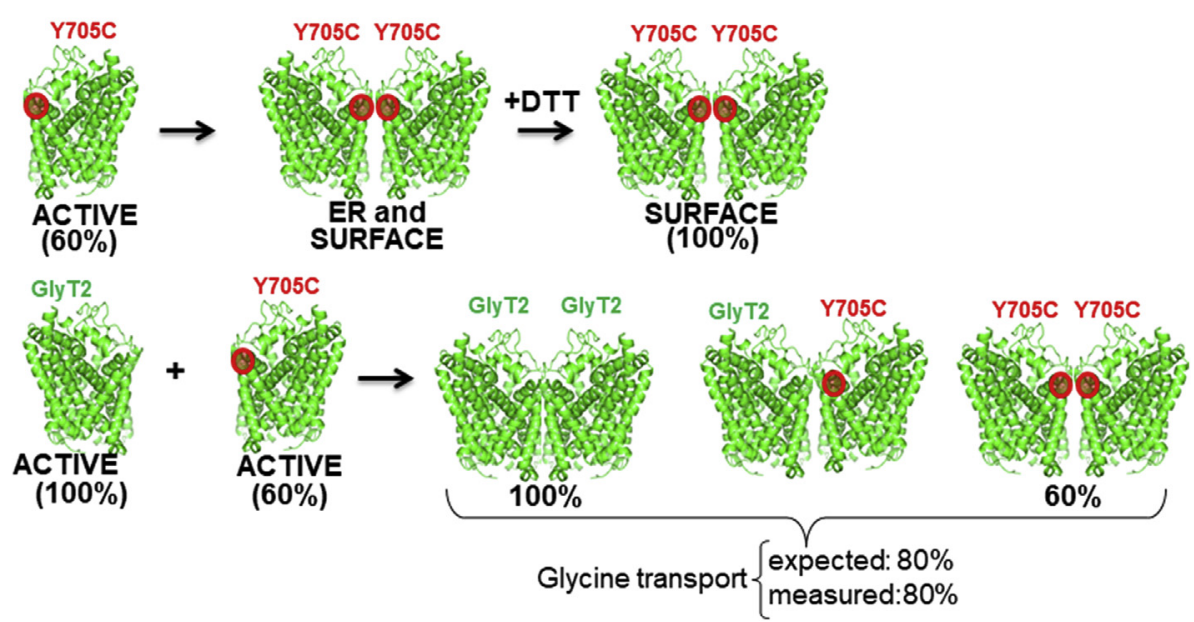

quaternary structure will unveil the correct oligomer assembly of the transporter and will provide valuable information for the study of mutations associated to hyperekplexia.

\section{Conclusion}

This short review contains the recent data on GlyT2 mutations involved in hyperekplexia. The neuronal GlyT2 is a crucial protein for glycine recycling in inhibitory glycinergic synapses and transporter loss of function abolishes glycinergic neurotransmission. Recessive mutations in GlyT2 gene (SLC6A5) are the second most common causes of hyperekplexia. The pathogenic mechanisms of two dominant mutations have been analyzed revealing different aspects of GlyT2 function can be affected in hyperekplexia. Dominance of two mutations supports the role of the quaternary structure of the transporter in physio-pathological aspects involving GlyT2.

\section{Acknowledgments}

This work was supported by the Spanish Ministerio de Economía y Competitividad (SAF2014-58045-R; SAF2017-84235-R). Institutional grants from the Fundación Ramón Areces and the Banco de Santander to the CBMSO are also acknowledged.

\section{References}

Anderluh, A., Hofmaier, T., Klotzsch, E., Kudlacek, O., Stockner, T., Sitte, H.H., Schütz, G.J., 2017. Direct PIP(2) binding mediates stable oligomer formation of the serotonin transporter. Nat. Commun. 8, 14089.

Andermann, F., Keene, D.L., Andermann, E., Quesney, L.F., 1980. Startle disease or hyperekplexia: further delineation of the syndrome. Brain 103, 985-997.

Aragon, C., Lopez-Corcuera, B., 2003. Structure, function and regulation of glycine neurotransporters. Eur. J. Pharmacol. 479, 249-262.

Aragon, C., Lopez-Corcuera, B., 2005. Glycine transporters: crucial roles of pharmacological interest revealed by gene deletion. Trends Pharmacol. Sci. 26, 283-286.

Armsen, W., Himmel, B., Betz, H., Eulenburg, V., 2007. The C-terminal PDZ-ligand motif of the neuronal glycine transporter GlyT2 is required for efficient synaptic localization. Mol. Cell. Neurosci. 36, 369-380.
Arribas-Gonzalez, E., Alonso-Torres, P., Aragon, C., Lopez-Corcuera, B., 2013. Calnexinassisted biogenesis of the neuronal glycine transporter 2 (GlyT2). PLoS One 8, e63230.

Arribas-Gonzalez, E., de Juan-Sanz, J., Aragon, C., Lopez-Corcuera, B., 2015. Molecular basis of the dominant negative effect of a glycine transporter 2 mutation associated with hyperekplexia. J. Biol. Chem. 290, 2150-2165.

Bakker, M.J., van Dijk, J.G., van den Maagdenberg, A.M., Tijssen, M.A., 2006. Startle syndromes. Lancet Neurol. 5, 513-524.

Barlowe, C., Helenius, A., 2016. Cargo capture and bulk Flow in the early secretory pathway. Annu. Rev. Cell Dev. Biol. 32, 197-222.

Bartholomaus, I., Milan-Lobo, L., Nicke, A., Dutertre, S., Hastrup, H., Jha, A., Gether, U., Sitte, H.H., Betz, H., Eulenburg, V., 2008. Glycine transporter dimers: evidence for occurrence in the plasma membrane. J. Biol. Chem. 283, 10978-10991.

Bogdanik, L.P., Chapman, H.D., Miers, K.E., Serreze, D.V., Burgess, R.W., 2012. A MusD retrotransposon insertion in the mouse Slc6a5 gene causes alterations in neuromuscular junction maturation and behavioral phenotypes. PLoS One 7, e30217.

Broer, S., Gether, U., 2012. The solute Carrier 6 family of transporters. Br. J. Pharmacol. $167,256-278$.

Carland, J.E. Thomas, M., Mostyn, S.N., Subramanian, N., O'Mara, M.L., Ryan, R.M. Vandenberg, R.J., 2018 Mar 21. Molecular determinants for substrate interactions with the Glycine transporter GlyT2. ACS Chem. Neurosci. 9 (3), 603-614.

Carta, E., Chung, S.K., James, V.M., Robinson, A., Gill, J.L., Remy, N., Vanbellinghen, J.F., Drew, C.J., Cagdas, S., Cameron, D., Cowan, F.M., Del Toro, M., Graham, G.E., Manzur, A.Y., Masri, A., Rivera, S., Scalais, E., Shiang, R., Sinclair, K., Stuart, C.A., Tijssen, M.A., Wise, G., Zuberi, S.M., Harvey, K., Pearce, B.R., Topf, M., Thomas, R.H., Supplisson, S., Rees, M.I., Harvey, R.J., 2012. Mutations in the GlyT2 gene (SLC6A5) are a second major cause of startle disease. J. Biol. Chem. 287, 28975-28985.

Claxton, D.P., Quick, M., Shi, L., de Carvalho, F.D., Weinstein, H., Javitch, J.A., McHaourab, H.S., 2010. Ion/substrate-dependent conformational dynamics of a bacterial homolog of neurotransmitter:sodium symporters. Nat. Struct. Mol. Biol. 17, $822-829$.

Coleman, J.A., Gouaux, E., 2018. Structural basis for recognition of diverse antidepressants by the human serotonin transporter. Nat. Struct. Mol. Biol. 25, 170-175.

Coleman, J.A., Green, E.M., Gouaux, E., 2016. X-ray structures and mechanism of the human serotonin transporter. Nature 532, 334-339.

Charlier, C., Coppieters, W., Rollin, F., Desmecht, D., Agerholm, J.S., Cambisano, N., Carta, E., Dardano, S., Dive, M., Fasquelle, C., Frennet, J.C., Hanset, R., Hubin, X., Jorgensen, C., Karim, L., Kent, M., Harvey, K., Pearce, B.R., Simon, P., Tama, N., Nie, H., Vandeputte, S., Lien, S., Longeri, M., Fredholm, M., Harvey, R.J., Georges, M., 2008. Highly effective SNP-based association mapping and management of recessive defects in livestock. Nat. Genet. 40, 449-454.

Chen, Z., Dillon, G.H., Huang, R., 2004. Molecular determinants of proton modulation of glycine receptors. J. Biol. Chem. 279, 876-883.

de Juan-Sanz, J., Nunez, E., Lopez-Corcuera, B., Aragon, C., 2013a. Constitutive endocytosis and turnover of the neuronal glycine transporter GlyT2 is dependent on 
ubiquitination of a C-terminal lysine cluster. PLoS One 8, e58863.

de Juan-Sanz, J., Nunez, E., Villarejo-Lopez, L., Perez-Hernandez, D., Rodriguez-Fraticelli, A.E., Lopez-Corcuera, B., Vazquez, J., Aragon, C., 2013b. Na +/K+-ATPase is a new interacting partner for the neuronal glycine transporter GlyT2 that downregulates its expression in vitro and in vivo. J. Neurosci. 33, 14269-14281.

de Juan-Sanz, J., Nunez, E., Zafra, F., Berrocal, M., Corbacho, I., Ibanez, I., ArribasGonzalez, E., Marcos, D., Lopez-Corcuera, B., Mata, A.M., Aragon, C., 2014. Presynaptic control of glycine transporter 2 (GlyT2) by physical and functional association with plasma membrane $\mathrm{Ca} 2+-\mathrm{ATPase}(\mathrm{PMCA})$ and $\mathrm{Na}+-\mathrm{Ca} 2+$ exchanger (NCX). J. Biol. Chem. 289, 34308-34324.

de Juan-Sanz, J., Zafra, F., Lopez-Corcuera, B., Aragon, C., 2011. Endocytosis of the neuronal glycine transporter GLYT2: role of membrane rafts and protein kinase Cdependent ubiquitination. Traffic 12, 1850-1867.

Drew, D., Boudker, O., 2016 Jun 2. Shared molecular mechanisms of membrane transporters. Annu. Rev. Biochem. 85, 543-572.

El-Kasaby, A., Just, H., Malle, E., Stolt-Bergner, P.C., Sitte, H.H., Freissmuth, M., Kudlacek, O., 2010. Mutations in the carboxyl-terminal SEC24 binding motif of the serotonin transporter impair folding of the transporter. J. Biol. Chem. 285, 39201-39210.

Ellgaard, L., Helenius, A., 2003. Quality control in the endoplasmic reticulum. Nat. Rev. Mol. Cell Biol. 4, 181-191.

Eulenburg, V., Becker, K., Gomeza, J., Schmitt, B., Becker, C.M., Betz, H., 2006. Mutations within the human GLYT2 (SLC6A5) gene associated with hyperekplexia. Biochem. Biophys. Res. Commun. 348, 400-405.

Farhan, H., Freissmuth, M., Sitte, H.H., 2006. Oligomerization of neurotransmitter transporters: a ticket from the endoplasmic reticulum to the plasma membrane. Handb. Exp. Pharmacol. 233-249.

Farhan, H., Korkhov, V.M., Paulitschke, V., Dorostkar, M.M., Scholze, P., Kudlacek, O. Freissmuth, M., Sitte, H.H., 2004. Two discontinuous segments in the carboxyl terminus are required for membrane targeting of the rat $\gamma$-aminobutyric acid Transporter-1 (GAT1). J. Biol. Chem. 279, 28553-28563.

Farhan, H., Reiterer, V., Korkhov, V.M., Schmid, J.A., Freissmuth, M., Sitte, H.H., 2007. Concentrative export from the endoplasmic reticulum of the $\gamma$-aminobutyric acid transporter 1 requires binding to SEC24D. J. Biol. Chem. 282, 7679-7689.

Fernandez-Sanchez, E., Diez-Guerra, F.J., Cubelos, B., Gimenez, C., Zafra, F., 2008 Mechanisms of endoplasmic-reticulum export of glycine transporter-1 (GLYT1). Biochem. J. 409, 669-681.

Fogarty, M.J., Kanjhan, R., Bellingham, M.C., Noakes, P.G., 2016. Glycinergic neurotransmission: a potent regulator of embryonic motor neuron dendritic morphology and synaptic plasticity. J. Neurosci. 36, 80-87.

Foster, E., Wildner, H., Tudeau, L., Haueter, S., Ralvenius, W.T., Jegen, M., Johannssen, H., Hosli, L., Haenraets, K., Ghanem, A., Conzelmann, K.K., Bosl, M., Zeilhofer, H.U., 2015. Targeted ablation, silencing, and activation establish glycinergic dorsal horn neurons as key components of a spinal gate for pain and itch. Neuron 85, 1289-1304.

Freissmuth, M., Stockner, T., Sucic, S., 2018. SLC6 transporter folding diseases and pharmacochaperoning. Handb. Exp. Pharmacol. 245, 249-270.

Gill, J.L., Capper, D., Vanbellinghen, J.-F., Chung, S.-K., Higgins, R.J., Rees, M.I., Shelton, G.D., Harvey, R.J., 2011. Startle disease in Irish wolfhounds associated with a microdeletion in the glycine transporter GlyT2 gene. Neurobiol. Dis. 43, 184-189.

Gimenez, C., Perez-Siles, G., Martinez-Villarreal, J., Arribas-Gonzalez, E., Jimenez, E., Nunez, E., de Juan-Sanz, J., Fernandez-Sanchez, E., Garcia-Tardon, N., Ibanez, I., Romanelli, V., Nevado, J., James, V.M., Topf, M., Chung, S.K., Thomas, R.H., Desviat, L.R., Aragon, C., Zafra, F., Rees, M.I., Lapunzina, P., Harvey, R.J., Lopez-Corcuera, B., 2012. A novel dominant hyperekplexia mutation Y705C alters trafficking and biochemical properties of the presynaptic glycine transporter GlyT2. J. Biol. Chem. 287, 28986-29002.

Gomeza, J., Ohno, K., Hulsmann, S., Armsen, W., Eulenburg, V., Richter, D.W., Laube, B., Betz, H., 2003. Deletion of the mouse glycine transporter 2 results in a hyperekplexia phenotype and postnatal lethality. Neuron 40, 797-806.

Guna, A., Hegde, R.S., 2018. Transmembrane domain recognition during membrane protein biogenesis and quality control. Curr. Biol. 28, R498-R511.

Hahn, M.K., Robertson, D., Blakely, R.D., 2003. A mutation in the human norepinephrine transporter gene (SLC6A2) associated with orthostatic intolerance disrupts surface expression of mutant and wild-type transporters. J. Neurosci. 23, 4470-4478.

Hammond, C., Braakman, I., Helenius, A., 1994. Role of N-linked oligosaccharide recognition, glucose trimming, and calnexin in glycoprotein folding and quality control. Proc. Natl. Acad. Sci. U. S. A. 91, 913-917.

Harvey, R.J., Topf, M., Harvey, K., Rees, M.I., 2008. The genetics of hyperekplexia: more than startle!. Trends Genet. 24, 439-447.

Harvey, R.J., Yee, B.K., 2013. Glycine transporters as novel therapeutic targets in schizophrenia, alcohol dependence and pain. Nat. Rev. Drug Discov. 12, 866-885.

Hirzel, K., Muller, U., Latal, A.T., Hulsmann, S., Grudzinska, J., Seeliger, M.W., Betz, H., Laube, B., 2006. Hyperekplexia phenotype of glycine receptor alpha1 subunit mutant mice identifies $\mathrm{Zn}(2+)$ as an essential endogenous modulator of glycinergic neurotransmission. Neuron 52, 679-690.

Imlach, W.L., 2017. New approaches to target glycinergic neurotransmission for the treatment of chronic pain. Pharmacol. Res. 116, 93-99.

Jardetzky, O., 1966. Simple allosteric model for membrane pumps. Nature 211, 969-970.

Krishtal, O.A., Osipchuk, Y.V., Shelest, T.N., Smirnoff, S.V., 1987. Rapid extracellular pH transients related to synaptic transmission in rat hippocampal slices. Brain Res. 436, 352-356.

Legendre, P., 2001. The glycinergic inhibitory synapse. Cell. Mol. Life Sci. 58, 760-793.

Lynch, J.W., 2004. Molecular structure and function of the glycine receptor chloride channel. Physiol. Rev. 84, 1051-1095.

Martinez-Maza, R., Poyatos, I., López-Corcuera, B., Núñez, E., Giménez, C., Zafra, F., Aragón, C., 2001 Jan 19. The role of N-glycosylation in transport to the plasma membrane and sorting of the neuronal glycine transporter GLYT2. J. Biol. Chem. 276 (3), 2168-2173.

Miller, E.A., Beilharz, T.H., Malkus, P.N., Lee, M.C., Hamamoto, S., Orci, L., Schekman, R., 2003. Multiple cargo binding sites on the COPII subunit Sec24p ensure capture of diverse membrane proteins into transport vesicles. Cell 114, 497-509.

Morrow, J.A., Collie, I.T., Dunbar, D.R., Walker, G.B., Shahid, M., Hill, D.R., 1998. Molecular cloning and functional expression of the human glycine transporter GlyT2 and chromosomal localisation of the gene in the human genome. FEBS Lett. 439, 334-340.

Nunez, E., Alonso-Torres, P., Fornes, A., Aragon, C., Lopez-Corcuera, B., 2008. The neuronal glycine transporter GLYT2 associates with membrane rafts: functional modulation by lipid environment. J. Neurochem.

Nunez, E., Aragon, C., 1994. Structural analysis and functional role of the carbohydrate component of glycine transporter. J. Biol. Chem. 269, 16920-16924.

Nunez, E., Perez-Siles, G., Rodenstein, L., Alonso-Torres, P., Zafra, F., Jimenez, E., Aragon, C., Lopez-Corcuera, B., 2009. Subcellular localization of the neuronal glycine transporter GLYT2 in brainstem. Traffic 10, 829-843.

Ozcan, L., Ergin, A.S., Lu, A., Chung, J., Sarkar, S., Nie, D., Myers Jr., M.G., Ozcan, U., 2009. Endoplasmic reticulum stress plays a central role in development of leptin resistance. Cell Metab. 9, 35-51.

Penmatsa, A., Gouaux, E., 2014. How LeuT shapes our understanding of the mechanisms of sodium-coupled neurotransmitter transporters. J. Physiol. 592, 863-869.

Penmatsa, A., Wang, K.H., Gouaux, E., 2013. X-ray structure of dopamine transporter elucidates antidepressant mechanism. Nature 503, 85-90.

Perez-Siles, G., Morreale, A., Leo-Macias, A., Pita, G., Ortiz, A.R., Aragon, C., LopezCorcuera, B., 2011. Molecular basis of the differential interaction with lithium of glycine transporters GLYT1 and GLYT2. J. Neurochem. 118, 195-204.

Perez-Siles, G., Nunez, E., Morreale, A., Jimenez, E., Leo-Macias, A., Pita, G., Cherubino, F., Sangaletti, R., Bossi, E., Ortiz, A.R., Aragon, C., Lopez-Corcuera, B., 2012. An aspartate residue in the external vestibule of GLYT2 (glycine transporter 2) controls cation access and transport coupling. Biochem. J. 442, 323-334.

Pourcho, R.G., Goebel, D.J., 1985. A combined Golgi and autoradiographic study of (3H) glycine-accumulating amacrine cells in the cat retina. J. Comp. Neurol. 233, $473-480$.

Rees, M.I., Harvey, K., Pearce, B.R., Chung, S.K., Duguid, I.C., Thomas, P., Beatty, S., Graham, G.E., Armstrong, L., Shiang, R., Abbott, K.J., Zuberi, S.M., Stephenson, J.B., Owen, M.J., Tijssen, M.A., van den Maagdenberg, A.M., Smart, T.G., Supplisson, S., Harvey, R.J., 2006. Mutations in the gene encoding GlyT2 (SLC6A5) define a presynaptic component of human startle disease. Nat. Genet. 38, 801-806.

Roux, M.J., Supplisson, S., 2000. Neuronal and glial glycine transporters have different stoichiometries. Neuron 25, 373-383.

Rudnick, G., Kramer, R., Blakely, R.D., Murphy, D.L., Verrey, F., 2014. The SLC6 transporters: perspectives on structure, functions, regulation, and models for transporter dysfunction. Pflugers Arch 466, 25-42.

Shipston, M.J., 2014. S-acylation dependent post-translational cross-talk regulates large conductance calcium- and voltage- activated potassium (BK) channels. Front. Physiol. $5,281$.

Sitte, H.H., Freissmuth, M., 2003. Oligomer formation by Na +-Cl-coupled neurotransmitter transporters. Eur. J. Pharmacol. 479, 229-236.

Subramanian, N., Scopelitti, A.J., Carland, J.E., Ryan, R.M., O'Mara, M.L., Vandenberg, R.J., 2016. Identification of a 3rd Na + binding site of the Glycine transporter, GlyT2. PLoS One 11, e0157583.

Thomas, R.H., Chung, S.-K., Wood, S.E., Cushion, T.D., Drew, C.J.G., Hammond, C.L., Vanbellinghen, J.-F., Mullins, J.G.L., Rees, M.I., 2013. Genotype-phenotype correlations in hyperekplexia: apnoeas, learning difficulties and speech delay. Brain 136, 3085-3095.

Tijssen, M.A.J., Rees, M.I., 2007 Jul 31. Hyperekplexia. [Updated 2012 Oct 4] In: Adam, M.P., Ardinger, H.H., Pagon, R.A. (Eds.), GeneReviews ${ }^{\circledR}$ [Internet]. University of Washington, Seattle (WA), pp. 1993-2018. Available from: https://www.ncbi.nlm. nih.gov/books/NBK1260\%.

Tijssen, M.A.J., Schoemaker, H.C., Edelbroek, P.J., Roos, R.A.C., Cohen, A.F., van Dijk, J.G., 1997. The effects of clonazepam and vigabatrin in hyperekplexia. J. Neurol. Sci. 149, 63-67.

van den Pol, A.N., Gorcs, T., 1988. Glycine and glycine receptor immunoreactivity in brain and spinal cord. J. Neurosci. 8, 472-492.

Vandenberg, R.J., Mostyn, S.N., Carland, J.E., Ryan, R.M., 2016. Glycine transporter2 inhibitors: getting the balance right. Neurochem. Int. 98, 89-93.

Vigevano, F., Di Capua, M., Bernardina, B., 1989. Startle disease: AN avoidable cause of sudden infant death. Lancet $333,216$.

Vuilleumier, P.H., Fritsche, R., Schliessbach, J., Schmitt, B., Arendt-Nielsen, L., Zeilhofer, H.U., Curatolo, M., 2018. Mutations affecting glycinergic neurotransmission in hyperekplexia increase pain sensitivity. Brain 141, 63-71.

Wenthold, R.J., Huie, D., Altschuler, R.A., Reeks, K.A., 1987. Glycine immunoreactivity localized in the cochlear nucleus and superior olivary complex. Neuroscience 22, 897-912.

Yamashita, A., Singh, S.K., Kawate, T., Jin, Y., Gouaux, E., 2005. Crystal structure of a bacterial homologue of $\mathrm{Na}+/ \mathrm{Cl}$-dependent neurotransmitter transporters. Nature 437, 215-223.

Zeilhofer, H.U., Acuña, M.A., Gingras, J., Yévenes, G.E., 2018. Glycine receptors and glycine transporters: targets for novel analgesics? Cell. Mol. Life Sci. 75, 447-465.

Zeppelin, T., Ladefoged, L.K., Sinning, S., Periole, X., Schiøtt, B., 2018. A direct interaction of cholesterol with the dopamine transporter prevents its out-to-inward transition. PLoS Comput. Biol. 14, e1005907.

Zomot, E., Gur, M., Bahar, I., 2015. Microseconds simulations reveal a new sodiumbinding site and the mechanism of sodium-coupled substrate uptake by LeuT. J. Biol. Chem. 290, 544-555. 